\title{
The use of desmopressin (DDAVP) for nocturia in women with multiple sclerosis
}

\author{
PAUL HILTON, KEITH HERTOGS, STUART L STANTON \\ From the Urodynamic Unit, Department of Obstetrics and Gynaecology, St. George's Hospital Medical \\ School, London, UK.
}

SUMMARY Sixteen women with multiple sclerosis who complained of nocturia completed a double-blind cross-over trial of Desmopressin (DDAVP) and placebo. Nocturia was reduced from a mean of 2.55 voids to 2.01 with placebo and to 1.28 with Desmopressin $(\mathrm{p}<0.01$, for the difference between placebo and Desmopressin). Side effects were minor, and equally distributed between treatment and placebo.

Desmopressin has been used to treat enuresis ${ }^{1}$ and nocturia ${ }^{2}$ in patients with a variety of urodynamic diagnoses. This trial was undertaken to follow-up our earlier observation that patients with multiple sclerosis benefited from Desmopressin. ${ }^{3}$

\section{Patients and methods}

Sixteen women with multiple sclerosis who complained of nocturia (waking from sleep to void on two or more occasions each night) were included in a double-blind crossover study comparing Desmopressin and placebo. The treatments were given at bed-time, in the form of an intranasal spray; the dose of Desmopressin was $20 \mathrm{mcg}$. The initial assessment included history, physical examination, a 7-day urinary diary, and a mid-stream urine specimen for culture. Grounds for exclusion were a history of impaired renal function, ischaemic heart disease, hypertension, or urinary infection. Treatment order was randomly allocated; nine patients took Desmopressin first, and seven took placebo first. During each treatment phase the patients kept a urinary diary. On the last day of each treatment phase a questionnaire of symptoms and sideeffects was completed, and measurements of blood pressure, plasma osmolality, electrolytes and urea, and early morning urine osmolality, made. Results were analysed using paired t-tests.

Address for reprint requests: Dr Paul Hilton, Newcastle General Hospital, Westgate Road, Newcastle-on-Tyne, NE4 6BE, UK

Recieved 15 February 1983 and in revised for from April 1983.

Accepted 27 March 1983.

\section{Results}

The only statistically significant differences between Desmopressin and placebo ( $p<0.01$ in each case; see table) were the early morning urine osmolality (significantly increased during Desmopressin treatment) and nocturia (significantly reduced during Desmopressin treatment).

\section{SUBJECTIVE BENEFITS}

Nine patients felt their nocturia improved on Desmopressin. One of these patients experienced improvement on placebo, and then further improvement on Desmopressin. No other patient noticed improvement on placebo. At the end of the trial eight patients wished to continue taking Desmopressin.

\section{SIDE-EFFECTS}

Headache was reported by three patients; in two the symptom was present during both phases of the trial, and in the third during the Desmporessin phase only. Nasal congestion was reported during the Desmopressin phase by one patient. Increased daytime urinary frequency was reported during the placebo phase by one patient. None of these patients stopped treatment, or found the symptoms severe enough to seek medical advice.

\section{Discussion}

Nocturia in patients with multiple sclerosis is a maniestation of their decreased functional bladder 
Table Clinical and biochemical results (mean \pm standard deviation)

\begin{tabular}{|c|c|c|c|c|c|}
\hline & Pre-treatment & Desmopressin & & Placebo & \\
\hline $\begin{array}{l}\text { Daytime frequency } \\
\text { Night-time frequency } \\
\text { Systolic blood pressure (mm Hg) } \\
\text { Diastolic blood pressure (mm Hg) } \\
\text { Early morning urine osmolality (mmol/kg) } \\
\text { Plasma osmolality (mmol/kg) } \\
\text { Plasma sodium (mmoll) } \\
\text { Plasma urea (mmol/l) }\end{array}$ & $\begin{array}{r}8.75 \pm 4.17 \\
2.55 \pm 0.75 \\
131.67 \pm 15.77 \\
79.67 \pm 9.72\end{array}$ & 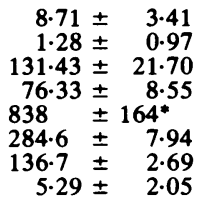 & $\begin{array}{l}\text { ns } \\
\text { ns } \\
\text { ns } \\
\text { ns } \\
\text { ns } \\
\text { ns }\end{array}$ & $\begin{array}{r}8 \cdot 61= \\
2 \cdot 01= \\
134 \cdot 29 \\
79 \cdot 64= \\
654 \\
283 \cdot 9= \\
138.7= \\
5 \cdot 4=\end{array}$ & $\begin{array}{lr} \pm & 2.46 \\
\pm & 0.86 \\
\pm & 19 \cdot 89 \\
\pm & 8 \cdot 87 \\
\pm & 223 \\
\pm & 9.59 \\
\pm & 2.61 \\
\pm & 1.96\end{array}$ \\
\hline
\end{tabular}

${ }^{*} \mathrm{p}<0.001 ;$ paired $t$ test

capacity. This may be due to detrusor instability, or to the presence of a residual urine volume. In patients with multiple sclerosis detrusor instability and voiding disorder commonly co-exist. There is no reason to suspect that Desmopressin improves the underlying bladder dysfunction; it acts by decreasing nocturnal urine production, and therefore increasing the time taken to reach bladder capacity. The magnitude of improvement in nocturia achieved in the current study corresponds well with the reduction in nocturnal urine output of $40 \%$ reported by Hilton and Stanton ${ }^{3}$ using the same dose of Desmopressin.

Before Desmopressin is considered for the treatment of nocturia, simple means of reducing nocturnal urine output (evening fluid restriction; avoiding bed-time drinks, particularly coffee and alcohol) should be tried. Several subjects needed help to use the nasal spray; the rhynile tube, supplied with commercially available Desmopressing, requires considerable manual dexterity, and many patients with multiple sclerosis will need help to use the drug correctly.
We acknowledge the support of Dr. Brian Donovan of Ferring Pharmaceuticals, who supplied the materials. We thank Action for Research into Multiple Sclerosis and The Multiple Sclerosis Society for publicising the study and encouraging their members to enrole. We thank Sister Lindsal Randall for nursing care of the patients, and the St. George's Hospital Pharmacy for dispensing the treatments.

\section{References}

' Ramsden PD, Hindmarsh JR, Bowditch JD, Price DA, Yeates WK. DDAVP for adult enuresis-a preliminary report. Proceedings of the IXth International Continence Society Meeting. Rome. Garroni. 1979;249-52.

${ }^{2}$ Taylor MC, Bates CP. DDAVP in the management of nocturnal frequency and enuresis due to bladder instability. Proceedings of the XIth International Continence Society Meeting. Lund. Skogs Trelleborg. 1981;144-5.

${ }^{3}$ Hilton P, Stanton SL. The use of Desmopressin in nocturnal urinary frequency in the female. $\mathrm{Br} J$ Urol 1982;54:252-5. 